\title{
Self-folding and aggregation of amyloid nanofibrils
}

\author{
Raffaella Paparcone*, Steven W. Cranford ${ }^{* * * *}$ and Markus J. Buehler****,
}

* Laboratory for Atomistic and Molecular Mechanics, Department of Civil and Environmental Engineering, Massachusetts Institute of Technology, 77 Massachusetts Ave. Room 1-235A\&B, Cambridge, MA, USA

** Center for Materials Science and Engineering, Massachusetts Institute of Technology, 77 Massachusetts Ave., Cambridge, MA, USA

${ }^{\dagger}$ Corresponding author, electronic address: mbuehler@MIT.EDU, Phone: +1-617-452-23750, Fax: $+1-617-324-4014$

Abstract: Amyloids are highly organized protein filaments, rich in beta-sheet secondary structures that self-assemble to form dense plaques in brain tissues affected by severe neurodegenerative disorders (e.g. Alzheimer's Disease). Identified as natural functional materials in bacteria, in addition to their remarkable mechanical properties, amyloids have also been proposed as a platform for novel biomaterials in nanotechnology applications including nanowires, liquid crystals, scaffolds and thin films. Despite recent progress in the understanding amyloid structure and behavior, the latent selfassembly mechanism and the underlying adhesion forces that drive the aggregation process remain poorly understood. On the basis of previous full atomistic simulations, here we report a simple coarse-grain model to analyze the competition between adhesive forces and elastic deformation of amyloid fibrils. We use simple model system to investigate self-assembly mechanisms of fibrils, focused on the formation of self-folded nanorackets and nanorings, and thereby address a critical issue in linking the biochemical (Angstrom) to micrometer scales relevant for larger-scale states of functional amyloid materials. We investigate the effect of varying the interfibril adhesion energy on the structure and stability of self-folded nanorackets and nanorings and demonstrate that such aggregated amyloid fibrils are stable in such states even when the fibril-fibril interaction is relatively weak, suggesting a strong propensity towards aggregation, given that the constituting amyloid fibril lengths exceed a critical fibril length-scale of several hundred nanometers. We further present a simple approach to directly determine the interfibril adhesion strength from geometric measures. In addition to providing insight into the physics of aggregation of amyloid fibrils our model enables the analysis of large-scale amyloid plaques and presents a new method for the estimation and engineering of the adhesive forces responsible of the self-assembly process of amyloid nanostructures, filling a 
gap that previously existed between full atomistic simulations of primarily ultra-short fibrils and much larger micrometer-scale amyloid aggregates. Via direct simulation of large-scale amyloid plaques consisting of hundreds of fibrils we demonstrate that the fibril length has a profound impact on their structure and mechanical properties, where critical fibril length-scale derived from our analysis defines the structure of amyloid plaques. A multi-scale modeling approach as used here, bridging the scales from Angstroms to micrometers, opens a wide range of possible nanotechnology applications by presenting a holistic framework that balances mechanical properties of individual fibrils, hierarchical self-assembly, and the adhesive forces determining their stability to facilitate the design of de novo amyloid materials.

Keywords: Amyloids; adhesion energy; mechanical properties; molecular dynamics simulations; coarse grain model; bending stiffness;

\section{Accepted for publication in: Nanoscale, DOI: 10.1039/CONR00840K}

\section{Introduction}

Plaques of $A \beta(1-40)$ amyloid fibrils are observed in the brain tissue affected by Alzheimer's Disease $(\mathrm{AD})^{1}$, a severe neurodegenerative disease. Along with many other biological materials - such as bone, silk, and cells - amyloids feature a hierarchical structure with geometric features at multiple scales $^{2}$. Amyloidogenic plaques derive from the aggregation of protofilaments composed of single fibrils that (at the atomistic level) are characterized by the stacking of sequence-wise identical layers, showing a twofold symmetry with respect to the growth axis $^{3}$ that results in their characteristic crossbeta geometry ${ }^{4}$ (Fig. 1A). The overall molecular structure of amyloid fibrils is stabilized by a dense network of H-bonds ${ }^{4}$, and features exceptional mechanical properties ${ }^{5,6}$ such as high stiffness ${ }^{5-8}$, selfassembling capacity $^{9-12}$, and remarkable stability even in adverse chemical environments ${ }^{13}$.

Hierarchical self-assembly of materials with such intriguing mechanical properties is a powerful developing tool for the creation of novel nanostructured materials ${ }^{14-16}$. There are examples of amyloids used as bionanomaterials in the form of nanowires ${ }^{10,17-19}$, scaffolds and (bio)templates ${ }^{10,20-}$ ${ }^{25}$, liquid crystals ${ }^{26}$, adhesives ${ }^{27}$ and films ${ }^{14}$. This wide range of applications is justified by the amyloid's remarkable mechanical and thermal stability and by their chemical properties that can be tuned via the introduction of additional elements, including enzymes, metal ions, fluorophores, biotin or cytochromes.

Recent results based on atomistic modelling provide a direct and quantitative link between molecular structure and mechanical behavior ${ }^{7,8}$, indicating a coupling between axial and twist deformation that 
facilitates mechanical stability. However, there is a dichotomy in approaches, where most quantitative experimental and theoretical studies have thus far focused on the scale of individual amyloid fibrils $^{5-7,28-32}$, other more qualitative experimental studies focused on amyloid aggregates (plaques) $^{14,33}$ at vastly larger scales, studying, for example, amyloid toxicity ${ }^{34-36}$. No quantitative, molecular based model exists to describe scales of hundreds of nanometers and micrometers, where particular key functional properties might emerge as amyloid fibrils form bundles and plaques (Figs. $1 \mathrm{~A}$ and 2A). A specific issue that remains poorly understood - albeit absolutely essential for the understanding of structure formation - is the competition between adhesive forces and elastic deformation of amyloid fibrils in the formation of bundles and plaques. This is a particularly intriguing question due to the great elastic stiffness (with Young's moduli exceeding 10-20 GPa), but rather weak (noncovalent) interactions between individual amyloid fibrils, posing a fundamental question of whether or not the weak interactions between fibrils are sufficiently strong to result in larger deformation and stable aggregation of amyloid fibrils into plaques. Here we address these issues by using a hierarchical multiscale approach in which results from atomistic simulations parameterize a coarse-grained representation of amyloid fibrils at scales approaching micrometers.

This paper is organized as follows: Section 2 (Materials and Methods) provides details of the atomistic simulation procedure used to estimate the adhesion energy and of the implementation of the mesoscale model. Section 3 reports a description of the results of atomistic simulations of 2-fibrils systems and of the behaviour of self-folded amyloid fibrils at the mesoscale, via the implementation of the coarse-grain model and the tuning of the adhesive forces driving the interaction between different fibrils, or part of the same fibril. In the same section we propose a connection between this study and experimental measurements on the basis of the observed geometry of folded amyloid fibrils. In the final Conclusion part (Section 4), the key contributions of this article are briefly highlighted.

\section{Materials and methods}

\subsection{Adhesion energy calculation from atomistic simulations}

Each layer and the corresponding 3D structure of the $A \beta(1-40)$ amyloid fibrils is provided by solid state NMR experimental data ${ }^{3}$, which allow to model only 32 of the 40 amino acids composing the sequence, since results from the first eight are disordered in experiments ${ }^{3}$. The structure of a 20 layer fibril is built following the model reported in Ref. 27 and considering the average configuration resulting from minimization and relaxation procedures ${ }^{37}$. Assuming that the interaction is 
homogenous along the length of the fibrils, all possible configurations of two amyloid fibrils interacting laterally are taken into account (Fig. 1B), where all cases are minimized and relaxed for approximately 5 nanoseconds at $300 \mathrm{~K}$ in implicit solvent, until convergence is reached. The followed simulation protocol and convergence criteria are the same as described in Ref. 24, which uses the CHARMM19 $9^{38}$ polar force field in conjunction with an effective Gaussian model for water ${ }^{39}$, and a time step of $1 \mathrm{fs}$. Each of the 2 fibril systems has been built imposing an interfibril distance sufficiently large to avoid any overlap between the lateral facing parts of the fibrils. The average distance between the two fibrils is $r_{\mathrm{adh}}=42.82 \AA$ (Fig. 1C), while the adhesion energy $\gamma_{0}$ has been estimated as $=4.39 \mathrm{kcal} /(\mathrm{mol}-\AA)$ (see next sections for details). Note that the approach used here to estimate the adhesion energy between fibrils has potential errors primarily due to limitations of the computational approach. Thus, the resulting adhesion energy is taken only as a base value that is varied over great ranges in the study reported here.

\subsection{Mesoscale model}

A series of full atomistic mechanical tests (tension and compression) ${ }^{7}$ provides a set of elastic parameters that, combined with the adhesion energy $\gamma_{0}$ and the average distance between two fibrils $r_{\text {adh }}$, allows us to develop a coarse-grained representation based on a hierarchical multiscale approach. In the coarse-grained representation three layers of the amyloid fibril are reduced to a single particle (bead) with a moment of inertia of $I=110,070.81 \times 10^{-40} \mathrm{~m}^{4}$, a cross-sectional area $A=1,414.32 \AA^{2}$ and a bond distance $r_{0}=12.72 \AA^{7}$. Assuming a homogenous distribution of mass, the mass of each particle represents the sum of the masses of all atoms composing the three layers, thus $m=30,940.98$ $\mathrm{g} / \mathrm{mol}$. The total energy of the system is evaluated as $E_{\mathrm{tot}}=E_{\mathrm{bond}}+E_{\text {angle }}+E_{\mathrm{adh}}$, where $E_{\text {bond }}$ is the energy stored in axial bond deformation, $E_{\text {angle }}$ is the energy contribution from bending and $E_{\text {adh }}$ describes adhesion. The bonds are evaluated on the basis of a series of harmonic potentials summing over all bonded pairs of beads, $E_{\mathrm{bond}}=\sum_{\text {bonds }} \varphi_{\mathrm{b}}(r)$. The stress-strain behavior of an A $\beta(1-40)$ amyloid fibril under tensile and compressive loading is non-linear and, in particular, both cases exhibit an increase of modulus from 2.34 GPa (for less than $0.168 \%$ strain in compression and $0.266 \%$ strain in tension), to $12.43 \mathrm{GPa}$ for compression and $18.05 \mathrm{GPa}$ for tension as reported in Ref. 13. Incorporating these results the bond energy function (accounting for nonlinearities) is expressed as:

$\varphi_{\mathrm{b}}=\frac{1}{2}\left\{\begin{array}{cc}k_{\mathrm{b}}^{s}\left(r_{\mathrm{stiff}}^{c}-r_{0}\right)^{2}+k_{\mathrm{b}}^{c}\left(r-r_{\mathrm{stiff}}^{c}\right)^{2}, \quad r \leq r_{\text {stiff }}^{c} \\ k_{\mathrm{b}}^{s}\left(r-r_{0}\right)^{2}, \quad r_{\text {stiff }}^{c}<r<r_{\text {stiff }}^{t} \\ k_{\mathrm{b}}^{s}\left(r_{\mathrm{stiff}}^{t}-r_{0}\right)^{2}+k_{\mathrm{b}}^{t}\left(r-r_{\mathrm{stiff}}^{t}\right)^{2}, \quad r \geq r_{\text {stiff }}^{t}\end{array}\right.$ 
Here $k_{\mathrm{b}}^{i}$ represents the spring constant relative to the strain range $i$ (c $=$ compression, $\mathrm{t}=$ tension, and $\mathrm{s}=$ small deformation). Based on the elastic moduli $E_{\mathrm{i}}$ identified in ${ }^{7}, k_{\mathrm{b}}^{i}=A E_{i} / r_{0}, k_{\mathrm{b}}^{\mathrm{c}}=198.84$ $\mathrm{kcal} /\left(\mathrm{mol}-\AA^{2}\right), k_{\mathrm{b}}^{\mathrm{s}}=37.43 \mathrm{kcal} /\left(\mathrm{mol}-\AA^{2}\right)$ and $k_{\mathrm{b}}^{\mathrm{t}}=288.74 \mathrm{kcal} /\left(\operatorname{mol}-\AA^{2}\right)$. A three-body potential is used to evaluate the bending energy contributions, given by the sum over all fibril angles, $E_{\text {angles }}=$ $\sum_{\text {angles }} \varphi_{\mathrm{a}}(\theta)$ and $\varphi_{\mathrm{a}}(\theta)=1 / 2 k_{\theta}\left(\theta-\theta_{0}\right)^{2}$ where $k_{\theta}=8,739.66 \mathrm{kcal} / \mathrm{mol}$ is the spring constant related to the bending stiffness, $\theta$ is the angle formed by three beads, and $\theta_{0}=180^{\circ}$ (equilibrium angle). Considering the elastic deformation energy of a beam with a point load, $k_{\theta} \propto E I / r_{0}$. We use a Lennard-Jones model to describe interfibril adhesion, $E_{\mathrm{adh}}=\sum_{\text {pairs }} \varphi_{\mathrm{LJ}}(r)$, and $\varphi_{\mathrm{LJ}}(r)=$ $4 \varepsilon\left([\sigma / r]^{12}-[\sigma / r]^{6}\right)$ with $\sigma=38.15 \AA$ and $\varepsilon=0.5 r_{0} \gamma_{0}=27.91 \mathrm{kcal} /(\mathrm{mol}-\AA)$ (following the approach put forth in Ref. 32). We note that the described mesoscale model is extremely simple by design, since the adopted bending potential contribution implies the isotropy of the fibrils bending stiffness, which can be considered correct only for fibrils longer than their helical pitch, which is approximately $120 \mathrm{~nm}$. The coarse-grain mesoscale model is implemented in LAMMPS ${ }^{40}$. An initial racket-type starting configuration is obtained by imposing a curvature to the central part of the fibril as reported in Fig. 2A (IV). A ring shaped state is obtained starting from a fibril in helix-like configuration. All structures are relaxed with an NVT ensemble at $300 \mathrm{~K}$ and equilibrated until structural convergence is reached.

\subsection{Plaque assembly}

To attain large multi-fibril systems, amyloid plaques are assembled in silico via a modified procedure developed for nanotube assembly akin to an approach described in a previous study ${ }^{41}$, wherein twelve sequential layers of 20 fibrils (240 fibrils total) are deposited on a substrate and equilibrated (see Fig. 4A; the same procedure was used to arrive at the plaque depicted in Fig. 1A). Each sequential layer is initially rotated by $60^{\circ}$ in-plane to facilitated entanglement and random orientation upon deposition. During assembly, the system is constrained by a cylindrical boundary with a diameter equal to the fibril length to facilitate fibril interaction. Upon each layer deposition, the assembly is equilibrated at $300 \mathrm{~K}$ for $2.5 \mathrm{~ns}$. After all layers have been deposited, boundary constraints are removed and the plaque is equilibrated at $300 \mathrm{~K}$ for an additional $10 \mathrm{~ns}$. The substrate is modeled by a Lennard-Jones wall potential, where $\varphi_{\text {wall }}(z)=\varepsilon_{w}\left(\frac{2}{15}\left[\sigma_{w} / z\right]^{9}-\left[\sigma_{w} / z\right]^{3}\right)$. Since it is not straightforward to directly link this strength of adsorption to experimental values, we choose parameters so as to mimic good 'anchoring' properties to the planar surface. Test cases indicate that deviations in the selected parameters ( $\varepsilon_{\mathrm{w}}$ and $\sigma_{\mathrm{w}}$ ) have a limited affect on the assembled structures. We note that the assembly 
procedure is implemented to obtain randomly entangled structures and not intended to mimic physical plaque growth.

\subsection{Computational nanoindentation}

We apply computational nanoindentation to characterize the contact stiffness of the assembled plaques (as described above following a similar approach as outlined in a previous study ${ }^{41}$ ). The indentation process itself is simple, where an indenter tip is pressed into the material and the forcedisplacement relationship is recorded (Fig. 4D). Indentation is simulated by the introduction of an indenter force field where a harmonic spherical indenter exerts a force of magnitude $F(r)=$ $K\left(r-R_{\text {ind }}\right)^{2}$, where $K$ is the specified force constant $(69.5 \mathrm{~N} / \mathrm{m}), r$ is the distance of a coarse-grain particle to the center of the indenter, and $R_{\text {ind }}$ is the radius of the indenter (50 nm). A constant indentation speed of $50 \mathrm{~m} \mathrm{~s}^{-1}$ was used. Both the force $(F)$ and depth $\left(d_{\text {ind }}\right)$ of the indenter tip are recorded during the simulation, resulting in the desired force-displacement relationship. Indentation is repeated several times at various locations in the plaque to account for random perturbations/variance and the average force-displacement curve is determined for each plaque.

Using the relation for contact stiffness ${ }^{42}, E_{\text {contact }}=S \sqrt{\pi / 4 A}$, where $A$ is the contact area of the indenter and $S$ is the calculated slope of the force-displacement curve. For the implemented spherical indenter, $A=2 \pi R_{\text {ind }} h_{\text {max }}$, where $R_{\text {ind }}$ is again the radius of the indenter, and $h_{\max }$ is the maximum depth of indentation (approx. $25 \mathrm{~nm}$ ). It is noted that the calculated contact modulus ( $\left.E_{\text {contact }}\right)$ does not necessarily reflect the Young's modulus of the amyloid plaques but provides a means of mechanical comparison between the cases considered.

\section{Results and discussion}

\subsection{Adhesion energies}

We first focus on quantifying fibril-fibril interactions, which derive from the combination of van der Waals, electrostatic and hydrophobic forces, here based on a full-atomistic model. We consider a system composed of two $\mathrm{A} \beta(1-40)$ amyloid fibrils as a model and determine the most stable arrangement and the corresponding adhesion energy. All possible configurations of two fibrils interacting laterally are taken into account (Fig. 1B). During the minimization and the subsequent relaxation (see Materials and Methods section for the details), the fibrils composing the systems reported in Fig 1B, rearrange thus optimizing the inter and intra-fibrils distances and interactions, but in the monitored time range, no further movement has been observed, nor switch of orientations of 
the fibrils. Further changes in the orientation of a single fibril could be investigated using different molecular simulation techniques (such as replica exchange approaches), which can circumvent the high energetic barriers separating eventual local minima. However, the fibrils composing the systems reported in Fig. 1B (Panels $I I I-V I$ ) tend to move apart during the relaxation, revealing the lack of a sufficiently large interfibril interaction due to the decreased contact area implied by the corresponding orientation.

For each case the total potential energy is averaged over the last 1 ns relaxation and the minimum energy value $\left(E_{2}\right)$ indicates the most favourable orientation (Fig. 1C and highlighted with a box in Fig. 1B). Similarly, for isolated fibrils, the average energy value $E_{1}$ is estimated over the last 1 ns relaxation of a pair of sufficiently isolated fibrils ${ }^{7,37}$. The adhesion energy per unit length $\gamma_{0}$ is defined as $\left(E_{2}-E_{1}\right) / l$ where $l=91.87 \AA$ (fibril length). The value of the adhesion energy in the most favourable orientation is $\gamma_{0}=4.39 \pm 0.32 \mathrm{kcal} /(\mathrm{mol}-\AA)$. The error on the value of the adhesion energy is estimated on the basis of the root mean square deviation relative to the last 1 ns relaxation calculated for both the energy values. The stability of this arrangement is mediated by a large contact area that promotes a great number of interactions among side chains. This arrangement also allows the system to maintain symmetry with respect to the fibril growth axis with twist periodicity in sync. However, the definition of the most stable configuration and of the corresponding value of $\gamma_{0}$, does not take into account the presence of the above mentioned disordered amino acids, which could drive differently the interaction and the arrangement of the fibrils, causing a variation of the estimated adhesion energy $\gamma_{0}$. The adhesion energy per unit area is defined as $\gamma_{\mathrm{A}}=\left(E_{2}-E_{1}\right) / A_{\mathrm{c}}$, where $A_{\mathrm{c}}=$ 2,965.61 $\AA^{2}$ is the contact area between two fibrils, estimated based on the length of 10 amino acids in the most inner part of the fibrils. This sequence is less affected by entropic perturbations and the variations of the corresponding contact area are reduced significantly. The adhesion energy per unit area is $\gamma_{\mathrm{A}}=0.14 \mathrm{kcal} /\left(\mathrm{mol}-\AA^{2}\right)$. This value is on the same order of magnitude of the adhesion energy of graphene, ranging from $0.22 \mathrm{kcal} /\left(\mathrm{mol}-\AA^{2}\right)^{43}$ to $0.37 \mathrm{kcal} /\left(\mathrm{mol}-\AA^{2}\right)^{44}$, or for the gecko spatulae that feature an adhesion energy of 0.07- $0.09 \mathrm{kcal} /\left(\mathrm{mol}-\AA^{2}\right)^{43}$.

\subsection{Analysis of the self-folded fibrils: nanorackets and nanorings}

Self-folded configurations of amyloid fibrils into racket-like and ring-shape forms at the mesoscale are considered here as simple models to develop fundamental insight into fibril aggregation mechanisms, by analyzing the effect of varying adhesion energy on resulting structures and stability. Despite the experimental evidences of self-folded amyloid fibrils, in particular of ring-shaped 
forms $^{45}$, to achieve a common structure of mechanical comparison, here the studied configurations are built imposing an initial defined curvature to the fibrils. These structures will be referred to as 'self-folded' for the purpose of this study.

Self-folding analysis has been used in earlier works applied to other systems including carbon nanotubes $^{46}$ and graphene ${ }^{44}$ and provides a simple yet powerful method to gain fundamental insight into self-assembly mechanisms of nanostructures. Here, we consider fibrils in racket- and ring-type shape, as reported in Fig. 2A ( $I V$ and $V$ ), respectively. For both configurations, the stability of the folded amyloid fibril with length $L$ is driven by the balance of the energy required to bend the fibril and the energy gained by the attractive forces, which are proportional to the bending stiffness, EI, and the adhesion energy, $\gamma_{0}$, respectively. A contact length, $L_{\mathrm{c}}$ is defined by the adhered segments. For the racket-type structure, the corresponding folded length is $2 L_{\mathrm{f}}=L-2 L_{\mathrm{c}}$ (Fig. 2A (II and $\left.I V\right)$ ). Here, we approximate the bending energy of a fibril using Euler-Bernoulli beam theory, where the bending energy per unit length is described as $U_{\text {bend }}=E I \int_{0}^{L_{\mathrm{f}}}\left(d^{2} u / d x^{2}\right)^{2} d x$, and where $u(x)$ is deflection. It has been shown that shear effects can be important for the deformation of such layered protein structures ${ }^{47}$, however, the fibrils studied here are considered long enough to neglect such contributions. The adhesion energy is expressed as $U_{\text {adhesion }}=\gamma L_{\mathrm{c}}$. Applying appropriate beam boundary conditions, and solving for

$$
U_{\text {total }}=U_{\text {bend }}-U_{\text {adhesion }}=2 \frac{\pi^{2}}{L_{\mathrm{f}}} E I+2 \gamma L_{\mathrm{f}}-\gamma L
$$

where $2 L_{\mathrm{f}}<L$. From the minimization of the total energy, we predict the adhesion energy of a fibril. Letting $d U / d L_{\mathrm{f}}=0$, we find:

$\gamma=E I\left(\frac{\pi}{L_{\mathrm{f}}}\right)^{2}$.

A similar derivation can be done for ring-type structures (Figs. 2A (III and V). Unlike racket-type folding, for overlapped ring structures, the adhered contact length, $L_{c}$, is also part of the folded length, approximating a circle of radius $r$. Thus, the fibril length is defined as $L=2 \pi r+L_{c}$. The elastic strain energy is defined as $U_{\text {ring }}=\frac{1}{2} \int_{0}^{L} E I \cdot \kappa^{2} d s$, where $\kappa=1 / r^{2}$, while the adhesion energy is defined as before $\left(\gamma L_{\mathrm{c}}\right)$. Allowing $U_{\text {total }}=U_{\text {ring }}-U_{\text {adhesion }}$, and minimizing with respect to the radius of the ring, it can be shown that:

$\gamma=\frac{E I \cdot L}{2 \pi \cdot r^{3}}$ 
Ring-shape structures show a dependence on the total length of the fibril $L$, which undergoes bending and contributes to both the adhesion and bending energy terms. Similar results have been shown in previous work for self-folding filamentous structures ${ }^{44,46,48,49}$ enabling a direct link between continuum theory and simulation or experiment. The simplified relations given in eq. (3) or eq. (4) can be used to compute the adhesion energy of an amyloid fibril if the folded length $L_{\mathrm{f}}$ can be measured from folded amyloidogenic structures (e.g. from experiment).

We now apply the coarse-grained model and consider amyloid fibrils with length $L \approx 255 \mathrm{~nm}$ and study the self-folding process to investigate the effect of adhesion energy on its stability in rackettype configurations. This analysis is motivated by the structure of amyloid plaques shown in Fig. $1 \mathrm{~A}(I)$ as well as the visualization in Fig. 2A that suggest that self-interactions of amyloid fibrils play an important role in plaque formation and as a result, in defining their structural and mechanical properties. We find that the fibril can be robustly folded into a stable racket- or ring-type shape, as demonstrated in Figs. 2B and C, for the reference adhesion energy $\gamma / \gamma_{0}=1$. This shows that in spite of the great stiffness of amyloid fibrils, the relatively weak interfibril interactions are sufficiently strong to drive the formation of a highly bent self-folded state. We systematically analyze the stability of the fibril in the racket-like and ring configuration by tuning the adhesion energy via variations of the ratio $\gamma / \gamma_{0}$. This approach allows us to quantify the effect of the adhesion energy on the geometry of the self-folded structure, and to effectively model changed environmental conditions such as variations in $\mathrm{pH}$, temperature, pressure and solvent that directly affect the interfibril adhesion (variations of these conditions have been experimentally analyzed by changing the solvent and by studying the variation of the fibril self-folding and aggregation rates ${ }^{50}$ ). The relaxed self-folded configurations are visualized in Figs. 2B and 2C, where snapshots of the relaxed amyloid fibrils correspond to different adhesion energies. We find that in both cases, higher values of the adhesion energy increase the contact length of the self-folded fibril, while it reduces if the adhesion energy is lowered. The values of contact and folded lengths $\left(L_{\mathrm{c}}\right.$ and $\left.L_{\mathrm{f}}\right)$ of the fibril in racket-type state are plotted over the adhesion energy in Fig. 3A.

Many experimental probes focus on simple mechanical assays (e.g. AFM bending tests), and, by extension, an inspection of folded structures provides a direct physical link between mechanical and energetic properties by using the results reported here. Specifically, the results reported here can be directly used by experimentalists to back-calculate the adhesion energy of the studied amyloid fibrils: geometric measurements of the rackety-type folded fibrils ("nanorackets”) obtained in the experiments provide evaluations of the contact $\left(L_{\mathrm{c}}\right)$ and free $\left(L_{\mathrm{f}}\right)$ lengths (Fig. 3B), and a bending 
stiffness can be determined from mechanical testing. Likewise, the radius, $r$, and contact length, $L_{\mathrm{c}}$, of ring-type structures ("nanorings”) can be measured. From this data, the corresponding adhesion energy can be estimated (Figs. 3C and 3D; eq. (3) and eq. (4)).

Moreover, from eq. (2), applying the condition that self-folding occurs when $U_{\text {total }}<0$ (i.e. the formation of folded structure is favorable), it can be seen that the critical total length to facilitate energetically stable self-folding is $L^{\text {crit,racket }}=4 \pi \sqrt{E I / \gamma}$. For the reference case considered here in which $\gamma=\gamma_{0}, L^{\text {crit }} \approx 115 \mathrm{~nm}$, which is smaller than the length of the fibril considered here and agrees with the observation in Fig. 2B that the self-folded state is stable. On the other hand, for the lowest adhesion energy modeled, $\gamma / \gamma_{0}=0.1$, we find $L^{\text {crit }} \approx 365 \mathrm{~nm}$ and thus we expect an unstable, self-straightening fibril, also in direct agreement with the findings shown in Fig. 2B. Specifically, the analysis predicts that a decrease of the adhesion energy does not result in the unfolding of the racketlike structure until a critical value is reached $\left(\gamma / \gamma_{0}<0.2\right.$, or an adhesion energy per unit area of 0.028 $\mathrm{kcal} /\left(\mathrm{mol}-\AA^{2}\right)$ and thus much smaller than van der Waals interactions as found in gecko spatulae). Below this level of adhesion, the folded length $\left(2 L_{\mathrm{f}}\right)$ required to achieve an energy minimum (as per eq. (2)) exceeds the total length of the fibril $(L)$ considered here, $L \approx 255 \mathrm{~nm}$. This result shows that folded amyloid fibrils are stable in folded states even when the fibril-fibril interaction is rather small, suggesting a strong propensity towards aggregation.

We also observe that increasing the adhesion energy results in a more prominent energy minimum, and thus more stable folded configurations as shown in Fig. 3C. The critical length to facilitate selffolding is dependent on both the adhesion energy and bending stiffness, which can vary according to specific experimental and environmental conditions. Similarly, we find for ring-type structures, $L^{\text {crit,ring }} \approx 3.67 \pi \sqrt{E I / \gamma}$. Thus, the formation of stable ring-type structures occurs at a slightly shorter fibril length. The analysis of the critical folding length and similarly, the diameter of amyloids folded into ring structures, provide a quantitative estimate of the length scale at which self-assembled amyloid structures emerge, resulting in a characteristic length-scale on the order of one hundred nanometers for the reference adhesion energy. This is an important finding that defines a scale at which mechanical properties of assemblies of amyloids are expected to show a significant change since the formation of entanglements based on racket-like structures and rings will induce novel deformation mechanisms. Specifically, assemblies of amyloid fibrils with short lengths at $L<L^{\text {crit }}$ are expected to feature more organized structures since bending of fibrils into complex entanglements 
due to interfibril adhesion is not possible, whereas assemblies of amyloids with $L \geq L^{\text {crit }}$ will feature a greater level of disorder.

To directly test this hypothesis and to potentially exploit this critical length-scale, we assemble and characterize large-scale multi-fibril assemblies (termed “plaques”). For the assembled plaques, we consider a constant adhesion energy $\gamma=\gamma_{0}$, such that $L^{\text {crit }} \approx 115 \mathrm{~nm}$. We specifically consider fibril constituents of three distinct lengths: $200 \mathrm{~nm}$ (above critical length), $100 \mathrm{~nm}$ (approximately critical length), and $50 \mathrm{~nm}$ (below critical length). Upon equilibration, the length of the fibrils has a direct impact on the final structure. As depicted in Fig. 4B, when $L=200 \mathrm{~nm}>L^{\text {crit }}$, the fibrils are more entangled and disordered. Loops, folds, and voids are clearly visible within the plaque structure, with no apparent preferred in-plane orientation. Conversely, when $L=100 \mathrm{~nm} \approx L^{\text {crit }}$, the fibrils tend to align along a common axis, while for $L=50 \mathrm{~nm}<L^{\text {crit }}$ the self-alignment results in a highly ordered in-plane configuration. It is noted that the assembly procedure is identical in all cases, and that it deposits layers in rotated orientations that induces entanglements and fibril folding. For short fibrils, self-alignment occurs upon equilibration and relaxation, and results in a more dense and ordered amyloid plaque. The mechanical analysis provided for fibril self-folding (Eq. (2) through Eq. (4)) cannot be applied directly to analyze fibrils within the plaque (due to multi-fibril interactions), but should be indicative of the length-scale in which plaques transition from an ordered to an entangled state. To quantify the state of disorder, we calculate the angle distribution along each fibril (i.e. multiple turns and folds within a fibril indicates a range of angle distributions, whereas aligned fibrils are relatively straight). For comparison, the deviation in angle from 180-degrees (i.e. perfectly linear) is calculated for each defined coarse-grain triple along all fibrils within the plaque and the frequency distribution plotted from 0 to 12 degrees (Fig. 4C). The peaks in the distribution are relative to the average curvature of the fibrils, and occur between $0^{\circ}-0.5^{\circ}$ for $50 \mathrm{~nm}$ fibrils, indicating an extremely high linearity, whereas $1.0^{\circ}-1.5^{\circ}$ for $100 \mathrm{~nm}$ fibrils and $1.5^{\circ}-2.0^{\circ}$ for the $200 \mathrm{~nm}$ fibrils. The relatively small angles are attributed to the stiffness of the fibrils, but peaks decrease in magnitude and the distribution of angles widens as the length of the fibrils increase as the fibril length increases. For or the current assembled plaques, the angle distributions are depicted in Fig. 4C, clearly illustrating a higher level of disorder when $L>L^{\text {crit }}$, as the range of calculated angles increases.

We also find that this changed structure has important implications on the mechanical properties, shown in Fig. 4D, which we assess via computational nanoindentation. The simulation of a nanoindentation is beneficial, as such investigations can be directly linked to actual physical 
experiments and providing a critical connection between empirical results and theoretical basis. Indentation is carried out to a depth of $25 \mathrm{~nm}$ for the $200 \mathrm{~nm}$ and $100 \mathrm{~nm}$ fibril plaques, while limited to $12.5 \mathrm{~nm}$ for the $50 \mathrm{~nm}$ fibril plaque as the smaller system becomes unstable. Further, for the smaller plaque system, we see a change in deformation mechanism under indentation. Due to the extremely high alignment of the fibril layers in the $50 \mathrm{~nm}$ fibril plaque, upon indentation, the loaded fibrils undergo "slip” along the adhesion plane, similar to slip of lattice planes in a crystalline material such as a metal. These slip events are reflected in regular drops of the force-displacement behavior (Fig. 4D), and occur approximately when the indentation depth reaches each sequential layer (3.6 nm to $4.0 \mathrm{~nm}$ ). The deviation from a purely elastic response inhibits the rigorous calculation of the contact modulus, but for a qualitative comparison the contact stiffness, $S$, is calculated from the final linear regime prior to slip. From the resulting force-displacement curves, we find that $E_{\text {contact }} \approx 0.67 \mathrm{GPa}$ for $L=200 \mathrm{~nm}, 1.88 \mathrm{GPa}$ for $L=100 \mathrm{~nm}$, and $3.26 \mathrm{GPa}$ for $L=50$ nm. Although the calculated contact modulus for the smallest plaque is an estimate due to the uncertainty in fitting, the force-displacement response clearly indicates higher contact stiffness. This immense decrease in stiffness (over $450 \%$ ) is attributed to the increased level of disorder within the larger system, and suggests the potential tunability of mechanical properties as a function of fibril length.

Aggregations of amyloid fibrils typically have lengths on the order of hundreds of nanometers, thus favoring the presence of such folds even with relatively small adhesion energies. Additionally, folding can occur between multiple fibrils under contact, as well the potential for multiple folds of ultra-long fibrils, facilitating growth and structural stability of aggregates and plaques. Such folding may result in complex mechanical entanglements, which, under various chemical environments that typically change the adhesion strength, maintain structural stability, serving to enhance the robustness of plaque formations. Further systematic investigation of adhesion strength variation, intermediate lengths scales, and the effect of mixed lengths is currently being studied.

The self-folding analysis reported here gives additional insight for the understanding of different shapes of amyloid fibrils at the mesoscale, such as the formation of end-to-end ring-shaped assemblies that have indeed been observed in the deposition of $\beta$-microglobuline amyloid fibrils or in the synthesis of insulin fibrils under pressure ${ }^{45}$. Other studies suggested that the inherently crowded environment of a living cell accelerates amyloid formation and aggregation ${ }^{51,52}$, while others relate the self assembly process to the presence of chaperone molecules ${ }^{53}$, or to increased peptide concentrations, altered $\mathrm{pH}$ and changed environmental conditions ${ }^{50}$. The results of our model propose 
a simple physical explanation of amyloid aggregation on the basis of adhesion between fibrils or parts of the same fibril.

\section{Conclusion}

The self-folding analysis of a single long fibril provides fundamental insight into the effect of fibrilfibril interactions (Figs. 2 and 3), which are responsible of the further aggregation of disordered and complex systems into amyloid plaques. Our computational and analytical multiscale study, bridging scales from Angstroms to micrometers, showed that the formation of these self-folded aggregates can be understood based on a balance of bending energy versus adhesion energy. The adhesion energy, as one of the key players driving the self-assembly and self-organization of amyloids into plaques, remains a key missing parameter in the range of physical properties associated with this class of protein materials. In this context, our analysis provides a simple and direct approach to calculate the adhesion energy based solely on the geometry of self-folded amyloid fibrils that can be easily measured from experiment. The estimation of the adhesion energy and of its variation according to the chemical environment represent an important step further to the understanding of amyloids selfassembly driving forces and for their effective utilization for the generation of new nanostructured materials and specifically for the applications in nanotechnology. Their performance in extreme mechanical, chemical or thermal conditions is highly dependent on the combination of the mechanical properties of both the individual fibrils and of the amyloid aggregates and the adhesive forces, which keep the self-assembled structures stable and promote the hierarchical organization of the material. Indeed, in a study of aggregation of a large number of amyloid fibrils into plaques we have directly demonstrated that the length of amyloid fibrils has important implications on the structure and mechanical properties of plaques (Fig. 4). Our data shows that for fibrils below a critical length highly ordered and stiff plaques emerge since the adhesion forces between fibrils are not sufficient to cause major bending. Conversely, for fibrils longer than a critical length highly entangled and disordered plaques are formed, which are significantly softer. This is explained by the fact that longer fibrils are increasingly stable in self-folded and ring-like geometries.

Acknowledgements: This research was supported by the Office of Naval Research (grant \# NN00014-08-1-0844) and NSF-MRSEC (grant \# DMR-0819762). Additional support from AROMURI is acknowledged (grant \# W911NF-09-1-0541).

\section{References}

1. $\quad$ F. Chiti and C. M. Dobson, Annu. Rev. Biochem., 2006, 75, 333-366.

2. M. J. Buehler and Y. C. Yung, Nat Mater, 2009, 8, 175-188. 
3. A. Petkova, Y. Ishii, J. Balbach, O. Anzutkin, R. Leapman, F. Delaglio and R. Tycko, P Natl Acad Sci USA, 2002, 99, $16742-16747$.

4. R. Nelson, M. R. Sawaya, M. Balbirnie, A. O. Madsen, C. Riekel, R. Grothe and D. Eisenberg, Nature, 2005, 435, 773-778.

5. T. P. Knowles, A. W. Fitzpatrick, S. Meehan, H. R. Mott, M. Vendruscolo, C. M. Dobson and M. E. Welland, Science, 2007, 318, 1900-1902.

6. J. Smith, T. Knowles, C. Dobson and M. Welland, P Natl Acad Sci USA, 2006, 43, 1580615811.

7. $\quad$ R. Paparcone, S. Keten and M. Buehler, J Biomech, 2010, 43, 1196-1201

8. $\quad$ Z. Xu, R. Paparcone and M. J. Buehler, Biophys J, 2010, 98, 2053-2062.

9. $\quad$ F. Gelain, D. Bottai, A. Vescovi and S. Zhang, PLoS ONE 1, 2006, e119.

10. T. Scheibel, R. Parthasarathy, G. Sawicki, X. Lin, H. Jaeger and S. Lindquist, P Natl Acad Sci USA, 2003, 100, 4527-4532.

11. S. Zhang, Nat. Biotechnol., 2003, 21, 1171-1178.

12. $\quad$ S. Zhang, T. Holmes, C. Lockshin and A. Rich, P Natl Acad Sci USA, 1993, 90, 3334-3338.

13. J. Zurdo, J. I. Guijarro and C. M. Dobson, J. Am. Chem. Soc., 2001, 123, 8141-8142.

14. T. Knowles, T. Oppenheim, A. Buell, D. Chirgadze and M. Welland, Nature Nanotechnol, 2010, 5, 204-207.

15. Y. Huang, X. Duan, Q. Wei, C. M. Lieber and Science 291, Science, 2001, 291, 630-633.

16. O. Ikkala and G. t. Brinke, Science, 2002, 295, 2407-2409.

17. $\quad$ Perutz M. F., Finch J. T., Berriman J. and L. A, Proc Natl Acad Sci U S A, 2002, 99, 55915595.

18. K. Lu, J. Jacob, P. Thiyagarajan, V. Conticello and D. Lynn, J Am Chem Soc, 2003, 125, 6391-6393.

19. M. Reches and E. Gazit, Science, 2003, 300, 625-627.

20. M. Reches and E. Gazit, J Nanosci Nanotechno, 2007, 7, 2239-2245.

21. O. Carny, D. Shalev and E. Gazit, Nano Lett, 2006, 6, 1594-1597.

22. Y. Song, S. Challa, C. Medforth, Y. Qiu, R. Watt, D. Pena, J. Miller, F. v. Swol and J. Shelnutt, Chem Commun, 2004, 1044-1045.

23. U. Baxa, V. Speransky, A. Steven and R. Wickner, P Natl Acad Sci USA, 2002, 99, 52535260.

24. A. Baldwin, R. Bader, J. Christodoulou, C. MacPhee, C. Dobson and P. Barker, J Am Chem Soc, 2006, 128, 2162-2163.

25. M. Yemini, M. Reches, J. Risphon and E. Gazit, Nano Lett, 2005, 5, 183-186.

26. A. Corrigan, C. Mueller and M. Krebs, J Am Chem Soc, 2006, 128, 14740-14741.

27. A. Mostaert, M. Higgins, T. Fukuma, F. Rindi and S. Jarvis, J Biol Phys, 2006, 32, 393-401.

28. I. Cherny and E. Gazit, Angew. Chem. Int. Ed., 2008, 47, 4062-4069.

29. A. Paravastu, R. Leapman, W.-M. Yau and R. Tycko, P Natl Acad Sci USA, 2008, 105, 18349-18354.

30. N. V. Buchete, R. Tycko and G. Hummer, J Mol Biol, 2005, 353, 804-821.

31. L. D. Mercato, G. Maruccio, P. P. Pompa, B. Bochicchio, A. M. Tamburro, R. Cingolani and R. Rinaldi, Biomacromolecules, 2008, 9, 796-803.

32. $\quad$ S. Guo and B. B. Akhremitchev, Biomacromolecules, 2006, 7, 1630-1636.

33. M. Buehler, Nature Nanotechnol, 2010, 5, 172-174.

34. G. Gouras, C. Almeida and R. Takahashi, Neurobiology of Aging, 2005, 26, 1235-1244.

35. S. Oddo, A. Caccamo, J. Shepherd, M. Murphy, T. Golde, R. Kayed, R. Metherate, M. Mattson, Y. Akbari and F. LaFerla, Neuron, 2003, 39, 409-421.

36. R. Friedrich, K. Tepper, R. Roenicke, M. Soom, M. Westermann, K. Reymann, C. Kaether and M. Faendrich, P Natl Acad Sci USA, 2010, 107, 1942-1947. 
37. R. Paparcone, J. Sanchez and M. J. Buehler, Journal of Computational and Theoretical Nanoscience., 2010, 7, 1279-1286.

38. B. R. Brooks, R. E. Bruccoleri, B. D. Olafson, D. J. States, S. Swaminathan and M. Karplus., J. Comp. Chem., 1983, 4, 187-217.

39. T. Lazardis and M. Karplus, Proteins: Structure, Function, and Bioinformatics, 1999, 35, 133-152.

40. $\quad$ S. Plimpton, Journal of Computational Physics, 1995, 117, 1-19.

41. S. W. Cranford and M. J. Buehler, Nanotechnology, 2010, 21, -.

42. G. M. Pharr, W. C. Oliver and F. R. Brotzen, Journal of Materials Research, 1992, 7, 613617.

43. Z. Zong, C.-L. Chen, M. Dokmeci and K.-t. Wan, Journal of Applied Physics, 2010, 107, 026104-026106.

44. S. Cranford, D. Sen and M. J. Buehler, Applied Physics Letters, 2009, 95, 122131.

45. R. Jansen, S. Grudzielanek, W. Dzwolak and R. Winter, J. Mol. Biol., 2004, 338.

46. M. J. Buehler, J. Mater. Res., 2006, 21, 2855-2869.

47. S. Keten, Z. P. Xu, B. Ihle and M. J. Buehler, Nature Materials, 2010, 9, 359-367.

48. W. Zhou, Y. Huang, B Liu, K. Hwang, J. Zuo, M. Buehler and H. Gao, Applied Physics Letters, 2007, 90, 073107-073109.

49. C. Py, P. Reverdy, L. Doppler, J. Bico, B. Roman and C. Baroud, Phys. Rev. Lett., 2007, 98, 156103.

50. $\quad$ C.-L. Shen and R. M. Murphy, Biophysical Journal, 1995, 69, 640-651.

51. A. Fulton, Cell, 1982, 30, 345-347.

52. P. LeDuc and R. Schwartz, Cell Biochemistry and Biophysics, 2007, 48, 16-31.

53. A. Kinjo and S. Takada, Biophys J, 2003, 85, 3521-3531.

54. W. Stine, K. Dahlgren, G. Krafft and M. LaDu, J Biol Chem, 2003, 278, 11612-11622. 


\section{Figures and figure captions}
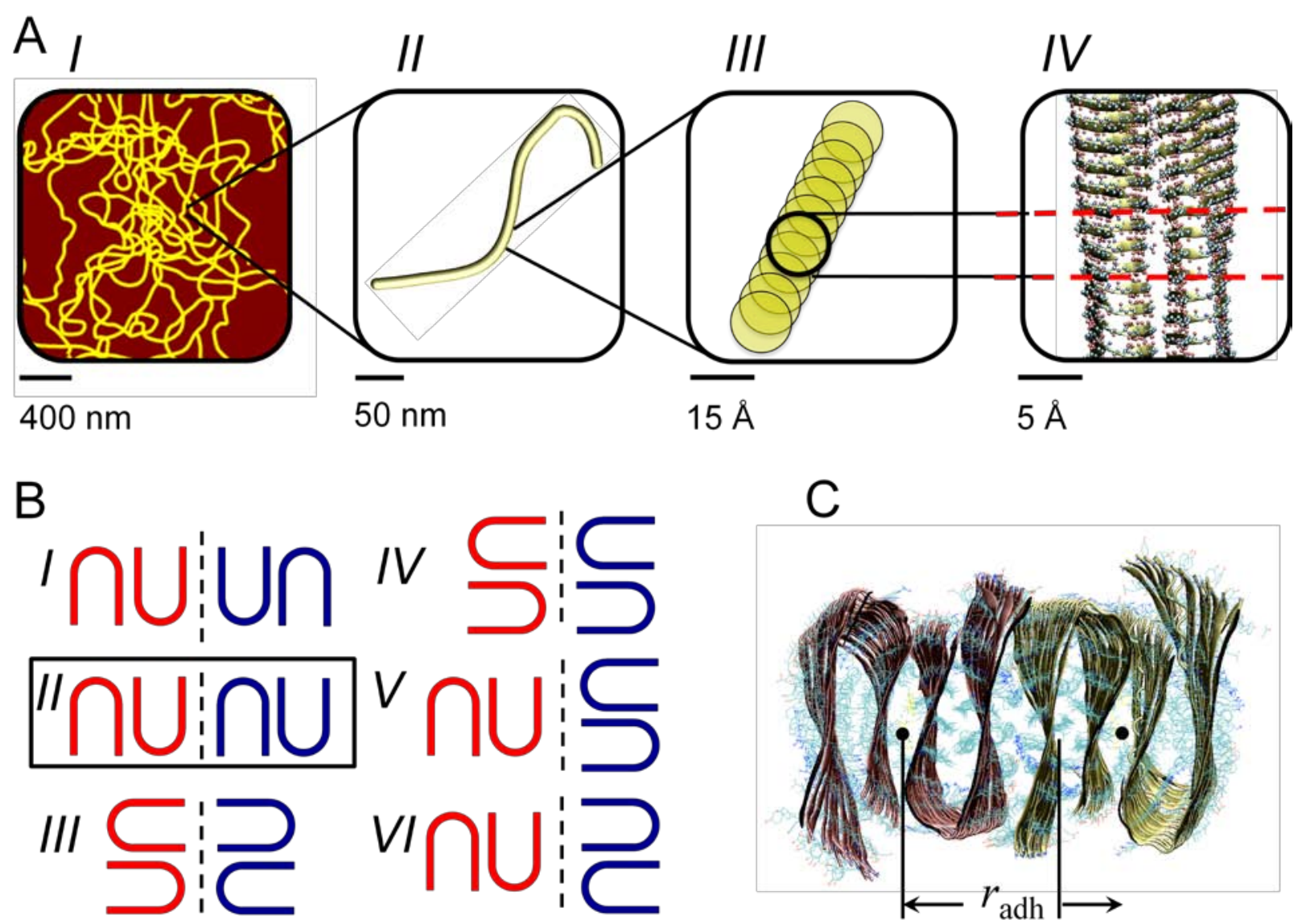

Fig. 1. Multiscale representation of $\mathrm{A} \beta(1-40)$ amyloid fibrils and evaluation of the fibril-fibril adhesion. A, Hierarchical structure of amyloid plaques from the Angstrom to micrometer lengthscales. (I) Schematic image of an amyloid plaque ${ }^{54}$, which appears as a complex ensemble of entangled fibers. (II) Schematic representation of a single amyloid fibril at the nanoscale. (III) Coarse-grain representation of the amyloid fibril. (IV) Representation of a two-fold symmetric A $\beta(1$ 40) amyloid fibril with atomistic details. B, Schematics of all possible arrangements of two amyloid fibrils. C, Relaxed system consisting of two beta-cross amyloid fibrils in the most stable configuration among all possible ones reported in panel B (II). The approach shown in panels B and $\mathrm{C}$ has a potential error associated with it (due to limitations of the computational approach); thus, the resulting adhesion energy is taken as a base value that is varied over great ranges in the study reported here. 


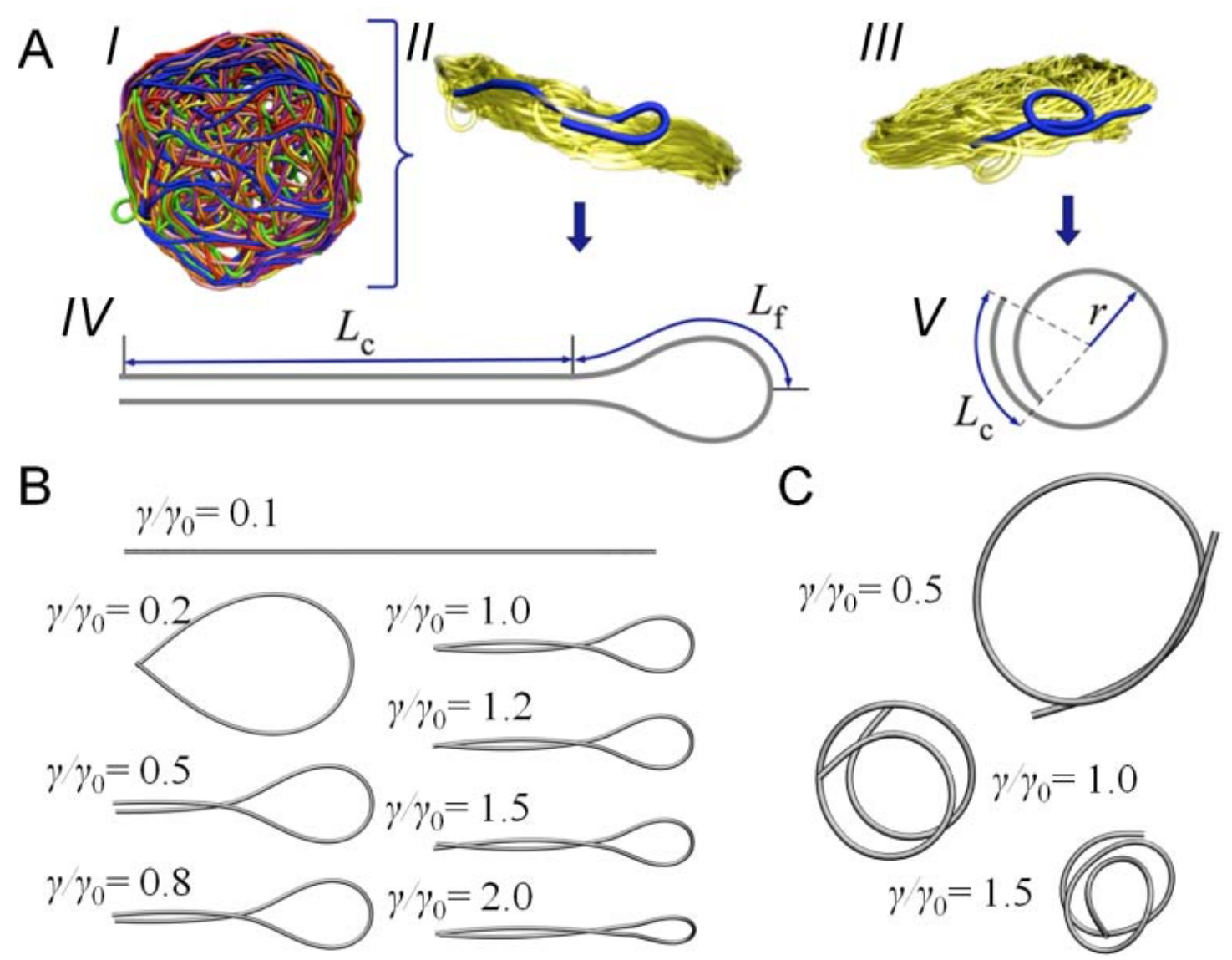

Fig. 2. Mesoscale representation of amyloid plaques and of fibrils in self-folded and ring-shaped states. A, Visualization of a complex amyloid plaque modeled with the coarse-grain model described here $(I)$. Examples of self-folded and ring-shaped amyloid fibrils identified within the larger plaque are depicted in subplots (II) and (III), respectively. Corresponding schematics of their geometry are shown in subplots (IV) and (V) with a definition of all key variables. B and C, Snapshots of amyloid fibrils in racket-like and ring-shaped states, obtained after relaxation at different adhesion energies (adhesion energies quantified by $\gamma / \gamma_{0}$ ). 

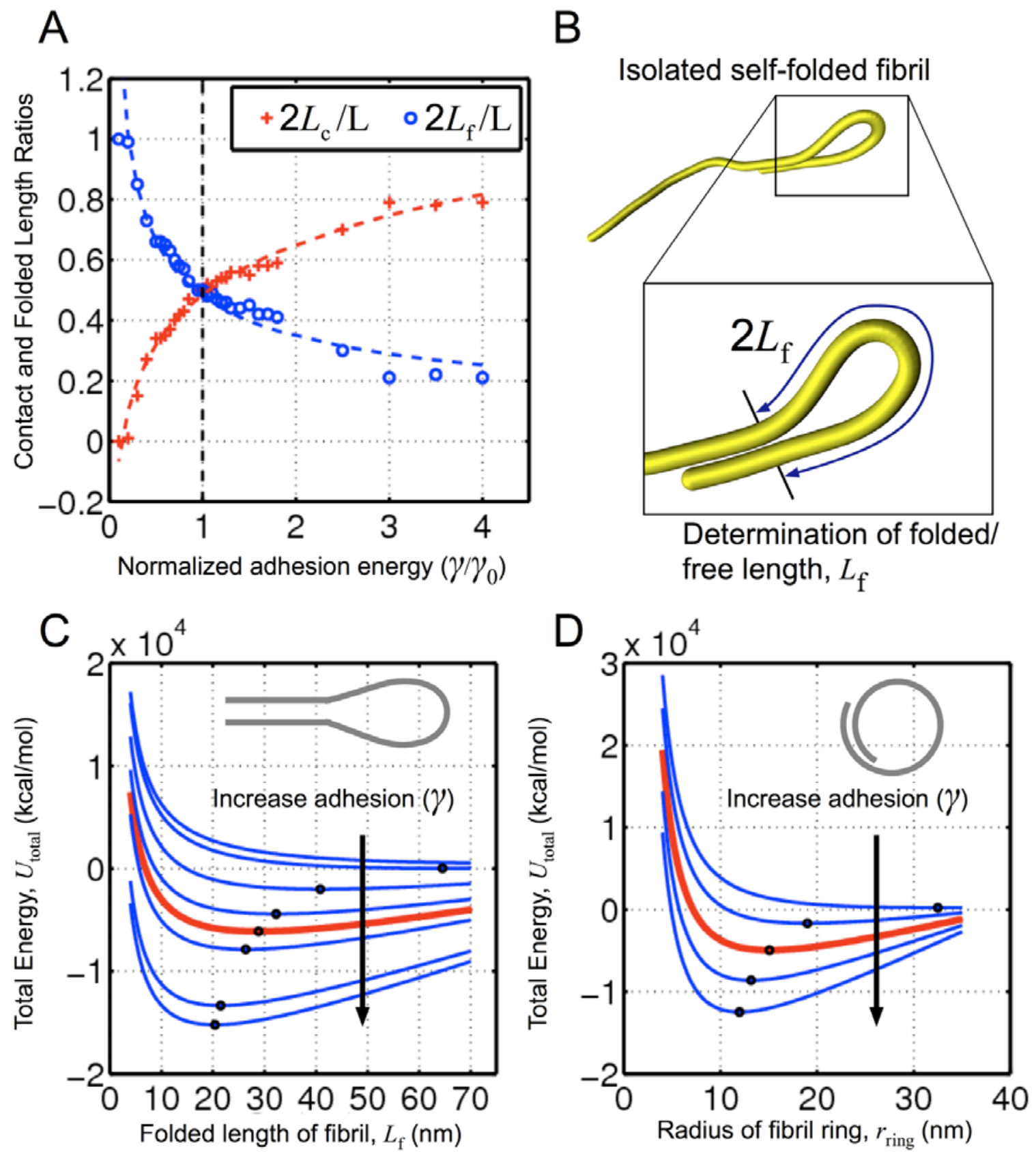

Fig. 3. Effect of the adhesion energy on amyloid fibrils in self folded and ring-shaped configurations. A, Contact length $\left(L_{\mathrm{c}}\right)$ and folded length $\left(L_{\mathrm{f}}\right)$ of an amyloid fibril in a racket-like configuration as a function of varied adhesion energy. B, Simple procedure to approximate the adhesion energy of amyloid fibrils via a geometric analysis of a stable folded structure with known bending stiffness, EI (see Eq. (3) and Eq. (4)). C and D, Total energy (Eq. (3) and Eq. (4)), as a function of folded length (in racket-type and ring-shaped state, respectively), demonstrating the decrease of the folded length required as a function of adhesion energy. 

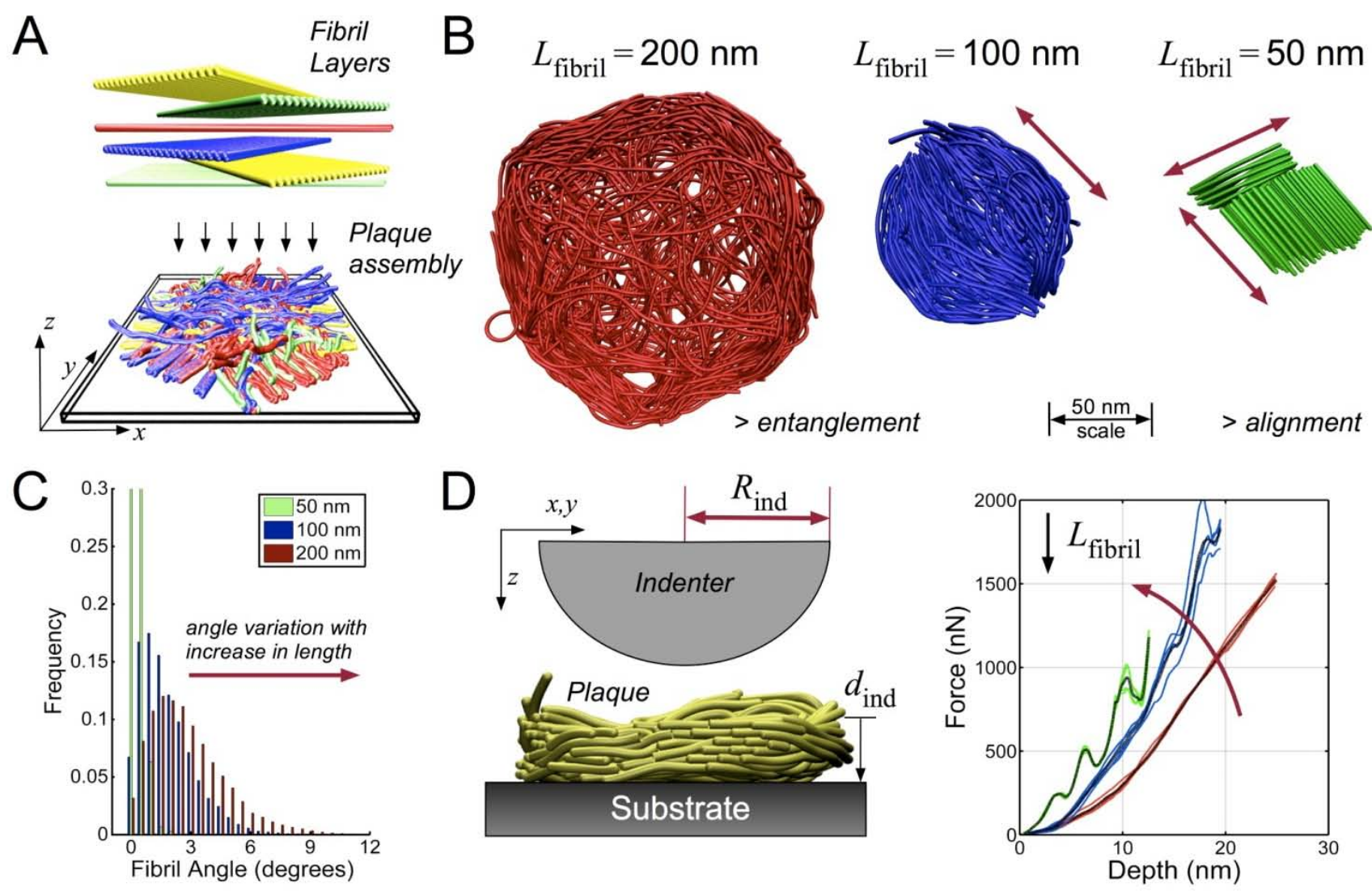

Fig. 4. Structural and mechanical investigation of large-scale amyloid plaques. A, Schematic of assembly procedure in which sequential layers of fibrils are deposited on a substrate (adapted from reference ${ }^{41}$ ); distinct layers of fibrils colored for clarity. B, Assembled plaques after relaxation and equilibration for fibril lengths greater than critical length $(200 \mathrm{~nm})$, close to critical length $(100 \mathrm{~nm})$, and less than critical length $(50 \mathrm{~nm})$. Plaques assembled with fibrils longer than the critical length maintain an entangled, disordered structure, with frequent occurrences of folds and loops, whereas plaques assembled with short fibrils undergo self-alignment under equilibration, resulting in a more ordered nanostructure. C, Quantification of disorder via angle distribution (frequency of angles among those defined by the coarse-grain fibrils). Here, the peak frequency is representative of average curvature of a single fibril. For the shortest fibrils $(50 \mathrm{~nm})$, over $90 \%$ of the angles are less than $1.0^{\circ}$, indicating relatively linear arrangements (frequency peaks omitted for clarity); for the 100 $\mathrm{nm}$ fibrils, over $75 \%$ of the angles are less than $2.5^{\circ}$, again indicating little curvature, whereas the longer fibrils (200 $\mathrm{nm}$ ) have a much broader range of angles due to the inherent folds and loops. D, Schematic and plot of computational nanoindentation of the $200 \mathrm{~nm}, 100 \mathrm{~nm}$, and $50 \mathrm{~nm}$ fibril plaques. Contact modulus (and contact stiffness) increases from approximately 0.67 GPa to $1.88 \mathrm{GPa}$ as the fibril length decreases from $200 \mathrm{~nm}$ to $100 \mathrm{~nm}$. Contact modulus approximated as 3.26 GPa for 50 nm fibril plaques, which undergo fibril layer “slip” under indentation (reflected by regular drops 
in force-displacement response). Variation of the length of the constituent fibrils illustrates the potential tunability of both the structural and mechanical properties of plaques and higher-order nanostructures. 